\title{
Intermittent versus continuous energy restriction on weight loss and cardiometabolic outcomes: a systematic review and meta-analysis of randomized controlled trials
}

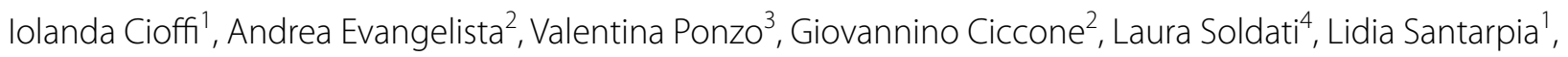
Franco Contaldo ${ }^{1}$, Fabrizio Pasanisi ${ }^{1}$, Ezio Ghigo ${ }^{3}$ and Simona $\mathrm{Bo}^{3^{*}}$ (D)

\begin{abstract}
Background: This systematic review and meta-analysis summarized the most recent evidence on the efficacy of intermittent energy restriction (IER) versus continuous energy restriction on weight-loss, body composition, blood pressure and other cardiometabolic risk factors.

Methods: Randomized controlled trials were systematically searched from MEDLINE, Cochrane Library, TRIP databases, EMBASE and CINAHL until May 2018. Effect sizes were expressed as weighted mean difference (WMD) and 95\% confidence intervals (Cl).

Results: Eleven trials were included (duration range 8-24 weeks). All selected intermittent regimens provided $\leq 25 \%$ of daily energy needs on "fast" days but differed for type of regimen (5:2 or other regimens) and/or dietary instructions given on the "feed" days (ad libitum energy versus balanced energy consumption). The intermittent approach determined a comparable weight-loss (WMD: $-0.61 \mathrm{~kg} ; 95 \% \mathrm{Cl}-1.70$ to $0.47 ; \mathrm{p}=0.87$ ) or percent weight loss (WMD: $-0.38 \%,-1.16$ to $0.40 ; p=0.34$ ) when compared to the continuous approach. A slight reduction in fasting insulin concentrations was evident with IER regimens (WMD $=-0.89 \mu \mathrm{U} / \mathrm{mL} ;-1.56$ to $-0.22 ; p=0.009$ ), but the clinical relevance of this result is uncertain. No between-arms differences in the other variables were found.
\end{abstract}

Conclusions: Both intermittent and continuous energy restriction achieved a comparable effect in promoting weight-loss and metabolic improvements. Long-term trials are needed to draw definitive conclusions.

Keywords: Continuous energy restriction, Intermittent energy restriction, Fasting glucose, Triglycerides, Weight loss

\section{Background}

In the last decade, much interest has been focused on dietary strategies that manipulate energy intake unconventionally, known as intermittent fasting or intermittent energy restriction (IER) [1-4]. This dietary approach has gained greater attention and popularity as a way for

\footnotetext{
*Correspondence: simona.bo@unito.it

${ }^{3}$ Department of Medical Sciences, University of Turin, c.so AM Dogliotti

14, 10126 Turin, Italy

Full list of author information is available at the end of the article
}

losing weight alternative to the conventional weight-loss diets, characterized by continuous (non-intermittent) energy restriction (CER). The two most popular forms of IER are: the 5:2 diet characterized by two consecutive or non-consecutive "fast" days and the alternate-day energy restriction, commonly called alternate-day fasting, alternate-day modified fasting, or every-other-day fasting, consisting of a "fast" day alternated with a "feed" day [5]. Commonly, during "fast" days, the energy intake is severely restricted, ranging from complete abstinence 
from foods to a daily maximum intake roughly corresponding to $75 \%$ energy restriction. Therefore, the term "fast" often does not involve a true complete abstinence from caloric intake. The term IER will be used to describe all intermittent energy-restricted/fasting regimens.

The time-restricted feeding [2, 6-9] and the very-lowcalorie or energy diets $[2,3]$ are other types of dietary interventions which were often included in previous systematic reviews and meta-analyses on IER. Indeed, in the former, individuals are allowed to eat within a specific range of time, thus, every day there is a period without food intake, varying from 12 to $21 \mathrm{~h}$ [10-12] (i.e. the Muslim Ramadan). On the other hand, there is no daily intermittency in a very-low-calorie-diet, although the overall energy intake may be similar to those of the IER regimens [13].

To the best of our knowledge, an overall evaluation of the impact of IER on multiple metabolic variables, on percent body fat changes, and on the effects of balanced versus ad libitum "feed" days, as well as on the benefits of the different "fasting" regimens is at present lacking.

The primary objective of this systematic review and meta-analysis was to update the efficacy of IER on weight loss, limiting the analyses to regimens which actually included a weekly intermittent energy restriction, i.e. from 1 up to 6 "fast days" per week. Furthermore, the impact of IER on fat mass (FM), fat free mass (FFM), arterial blood pressure (BP) and other cardiometabolic risk factors was assessed. The effects of IER according to the specific type of nutritional regimen on all these outcomes were evaluated too.

\section{Materials and methods}

We followed the Preferred Reporting Items for Systematic Reviews and Meta-Analyses (PRISMA) guidelines in the reporting of this study [14].

\section{Search strategy}

The following electronic databases were queried using a combination of search terms until the 31th of May 2018: PubMed (National Library of Medicine), the TRIP database, the Cochrane Library, EMBASE, and Cumulative Index to Nursing and Allied Health Literature (CINAHL). The construction of the search strategy was performed using database specific subject headings and keywords. Both medical subject headings $(\mathrm{MeSH})$ and free text search terms were employed. Restrictions to human studies were placed.

The search terms included combinations of "intermittent fasting" or "alternate day fasting" or "intermittent energy restriction" or "periodic fasting", and weight loss, weight gain, obesity, weight, fat mass, blood pressure, blood glucose, insulin, insulin-resistance, insulin sensitivity, glycated hemoglobin A1c (HbA1c), type 2 diabetes mellitus (T2DM), cholesterol, and triglycerides (free-term and MESH as possible) (Additional file 1). These search strategies were implemented by hand searching the references of all the included studies and systematic reviews on the field.

\section{Study selection}

We included studies with the following characteristics: (1) randomized controlled trials (RCTs); (2) a detailed description of the IER regimen; (3) $75 \%$ of energy restriction on "fast" days, with a maximum cut-off of $500 / 660 \mathrm{kcal} /$ day for females/males, respectively; (4) weekly intermittency of energy restriction (from 1 up to 6 "fast" days per week); (5) trial duration $>4$ weeks; (6) containing as comparator a group on a CER regimen and (7) including changes in body weight or percent body weight as one of the study's outcome.

We excluded studies with the following characteristics: (i) uncontrolled trials or study design other than RCTs; (ii) studies not including body weight as an outcome and/or lacking sufficient information on weight change; (iii) including time restricted feeding intervention; (iv) reporting very-low-calorie or fasting regimens for $>6$ days consecutive/week; and (v) providing $>500$ $660 \mathrm{kcal} /$ day or not reporting the amount of calorie prescribed on "fast" days.

In trials with multiple interventional arms (i.e. exercise arm, intervention arm with specific diets), the IER and the CER arms were considered, while other arms were not analyzed, since out of the scope of this review.

Two authors (IC, SB) separately screened abstracts for their inclusion or exclusion; retrieving full text articles from potentially relevant abstracts. Any discrepancy about inclusion was resolved by discussing with a third author (AE).

\section{Outcomes}

The primary outcome of the review was evaluating changes in body weight or in percent body weight. Secondary outcomes were: changes in body mass index (BMI), waist circumference, FM, FFM, arterial BP, and the blood values of fasting glucose and insulin, insulin resistance, insulin sensitivity, HbA1c, total cholesterol, HDL- and LDL-cholesterol, and triglycerides. The changes of these outcomes according to the specific type of IER regimen were also evaluated.

\section{Data collection and extraction}

From each included study, the following information were extracted (1) first author name and year of publication; (2) study design; (3) inclusion criteria of 
participants; (4) trial duration; (5) number of subjects enrolled in each arm; (6) type of dietary intervention; (7) age, gender, BMI of participants; (8) body composition (FM and FFM); (9) systolic (SBP) and diastolic blood pressure (DBP); (10) blood concentrations of fasting glucose, HbA1c, insulin, total cholesterol, HDL-cholesterol, LDL-cholesterol, and triglycerides; (11) Homeostasis Model Assessment-Insulin Resistance (HOMA-IR) and insulin-sensitivity index $(\mathrm{Si})$.

\section{Risk of bias assessment}

All studies were independently assessed by two authors (IC, SB) using the "Risk of bias" tool developed by the Cochrane Collaboration for RCTs [15]. The items used for the assessment of each study were the following: adequacy of sequence generation, allocation concealment, blinding, addressing of dropouts (incomplete outcome data), selective outcome reporting, and other potential sources of bias. A judgment of "L" indicated low risk of bias, " $\mathrm{H}$ " indicated high risk of bias, and "unclear" indicated an unclear/unknown risk of bias. The possible disagreements were resolved by consensus, or with consultation with a third author (AE).

\section{Data synthesis}

Data synthesis was performed only for the outcomes which were reported by $>3$ trials.

The pooled effect sizes were expressed as weighted mean differences (WMD) and 95\% confidence interval (CI) between IER and CER arms of the mean outcome values measured at the end of follow-up.

The mean difference of changes from baseline was estimated for each study on the basis of reported baseline and follow-up measurements. If the standard deviation for change from baseline was not reported, we imputed missing values assuming a within-patient correlation from baseline to follow-up measurements of 0.8 as suggested in the Cochrane handbook [16]. When betweenarms mean differences on change from baseline were already estimated [17], those data were included. For the relative weight change from baseline, the non-reported standard deviations were imputed using the mean standard deviation of the available studies.

Random-effects models were applied to provide a summary estimate.

Inter-study heterogeneity was assessed using Cochrane $\mathrm{Q}$ statistic and quantified by $\mathrm{I}^{2}$ test [18].

Subgroup analyses for all outcomes were performed based on the different dietary regimen of the "feed" days (balanced vs. ad libitum food intake) and the effects of the different regimens of "fasting" (5:2 vs. the other regimens). Weighting of studies was done using generic inverse variance method.

In order to evaluate the influence of each study on the overall effect size, sensitivity analysis was conducted using the one-study remove (leave-one-out) approach.

Potential publication bias was explored using visual inspection funnel plot asymmetry and Egger's weighted regression tests.

Meta-analyses were performed by using the Stata Metan package (Stata Statistical Software, Release 13; StataCorp LP, College Station, TX); meta-regressions and Egger's weighted regression tests for publication bias were performed using the metafor package (version 1.97) for R (version 3.1.2, R Foundation for Statistical Computing, Vienna, Austria).

\section{Results \\ Included studies}

The initial literature search identified 8577 records. After removing duplicates, 6943 records were screened, and, after excluding articles not meeting the inclusion criteria, 94 records were assessed for eligibility. After further analysis and quality assessment, a total of 11 studies were selected for the systematic review and meta-analysis (Fig. 1). All studies identified were RCTs, reporting an IER arm and a CER arm comparison; the corresponding details are shown in Table 1. Data relative to participants involved in exercise-only arms [19] or in high-protein dietary intervention [20] were not considered, because not pertinent to the aims of the study.

\section{Characteristics of the studies}

The total number of subjects included in the present analysis was 630 at enrolment. During the course of the trials, 102 patients dropped out. Drop-out rates ranged from about $2 \%$ [21] to $38 \%$ for IER arms [22] and from $0 \%$ [23] to 50\% [22] for CER. The number of participants analyzed at the end of the RCTs was 528 .

There was a greater number of women among participants, with the exception of 3 studies with a balanced number between men and women [21, 22, 24] and 1 enrolling only men [23]. Participants were individuals with overweight/obesity; in 2 RCTs patients with T2DM were selected $[23,25]$, and in 1 RCT patients with multiple dysmetabolic conditions were enrolled [21]. In all RCTs except for $2[23,25]$, participants with a stable weight before the beginning of the study, without history of bariatric surgery, and without drugs impacting on weight or the other study outcomes, were studied.

Trials were performed in UK [20, 22, 26], in USA [17, $19,25,27]$, in Australia [23, 24], and Norway [21, 28]. The duration of the studies ranged from 8 weeks [27] to 24 weeks $[17,21,23,26]$. 


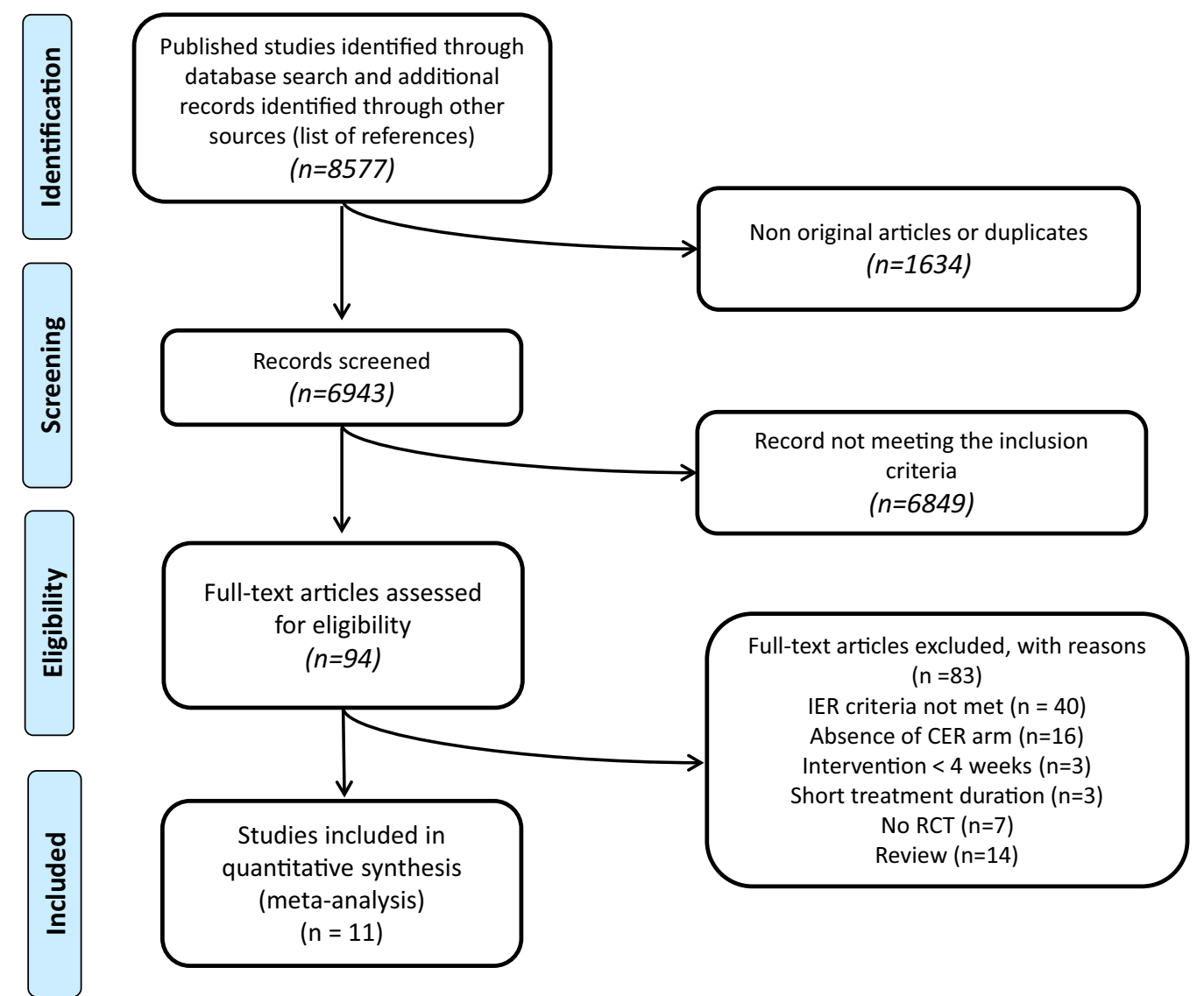

Fig. 1 Flow of the study

\section{Dietary intervention}

Four studies prescribed alternating "fast" and "feed" days $[17,19,27,28]$. Six studies used 2 "fast" days and 5 "feed" days per week (5:2 diet) [21-24, 26]. In 1 RCT, 5 consecutives "fast" days were prescribed before a 1 "fast" day/week regimen per 15 weeks in the IER arm, while the other arm ( 5 "fast" days every 5 weeks) was not considered, since no intermittence within the same week was present [25]. On "fast" days, diets provided a maximum of $660 \mathrm{kcal} /$ day. In 2 studies, participants were instructed to consume their meals between 12:00 p.m. and 2:00 p.m. on "fast" days to ensure that subjects underwent the same duration of fasting $[17,19]$. In 4 studies, meals of "fast" days were partially $[17,25]$ or totally supplied $[19,27]$. In 1 study, a commercially available very-low energy formula-based food was assigned in the "fast" days [22].

On "feed" days, 6 studies prescribed healthy and balanced eating pattern, according to the energy requirements [17, 20, 22, 25, 26, 28], 4 allowed for ad libitum food intake based on the participants' usual eating
[19, 21-24] and 1 provided a diet based on the energy requirements but allowing the access to 5-7 optional food modules (200 kcal each) [27]. In the comparator arms, energy was restricted by approximately $25 \%$ of the daily energy requirements in all studies (CER arms).

\section{Dietary compliance and energy intake assessment}

Six studies specifically assessed the compliance to the diet and the overall energy intake in both arms by filling 7 -day food records at different time points [17, 20-22, 26, 28]. In 1 study, dieticians evaluated adherence by using patients' self-recorded dietary diaries and diet histories taken during their dietetic appointments [23]. Either similar adherence between IER and CER [20, 21, 23, 26, 28], a lower [17] or a higher [22] adherence in the IER arms were reported. Adherence to the recommendations in the IER arms ranged from 64\% [26] to 93\% [22] at the end of the RCTs, but data were difficult to compare because of their incompleteness and the different methods employed to evaluate the compliance. 


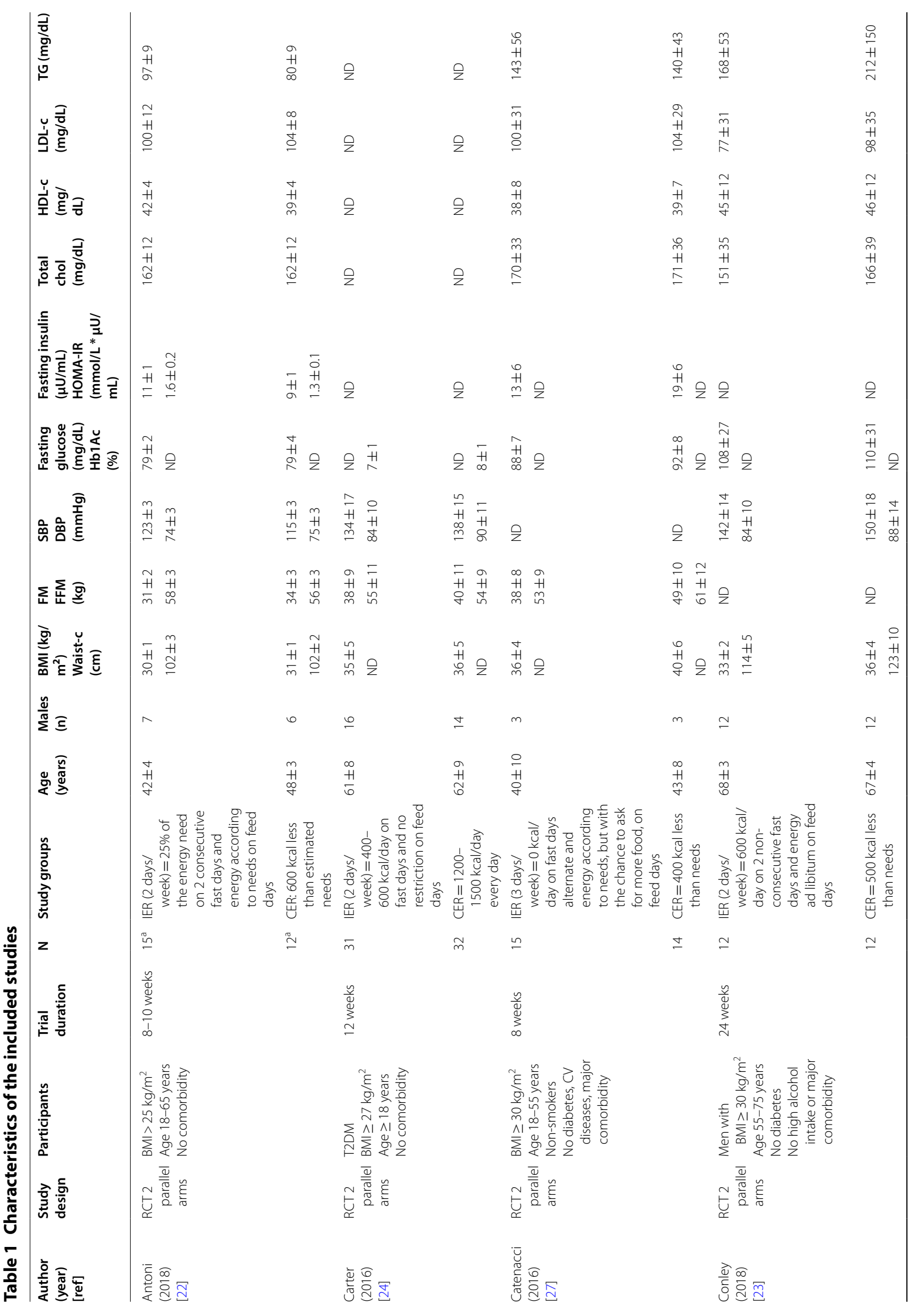




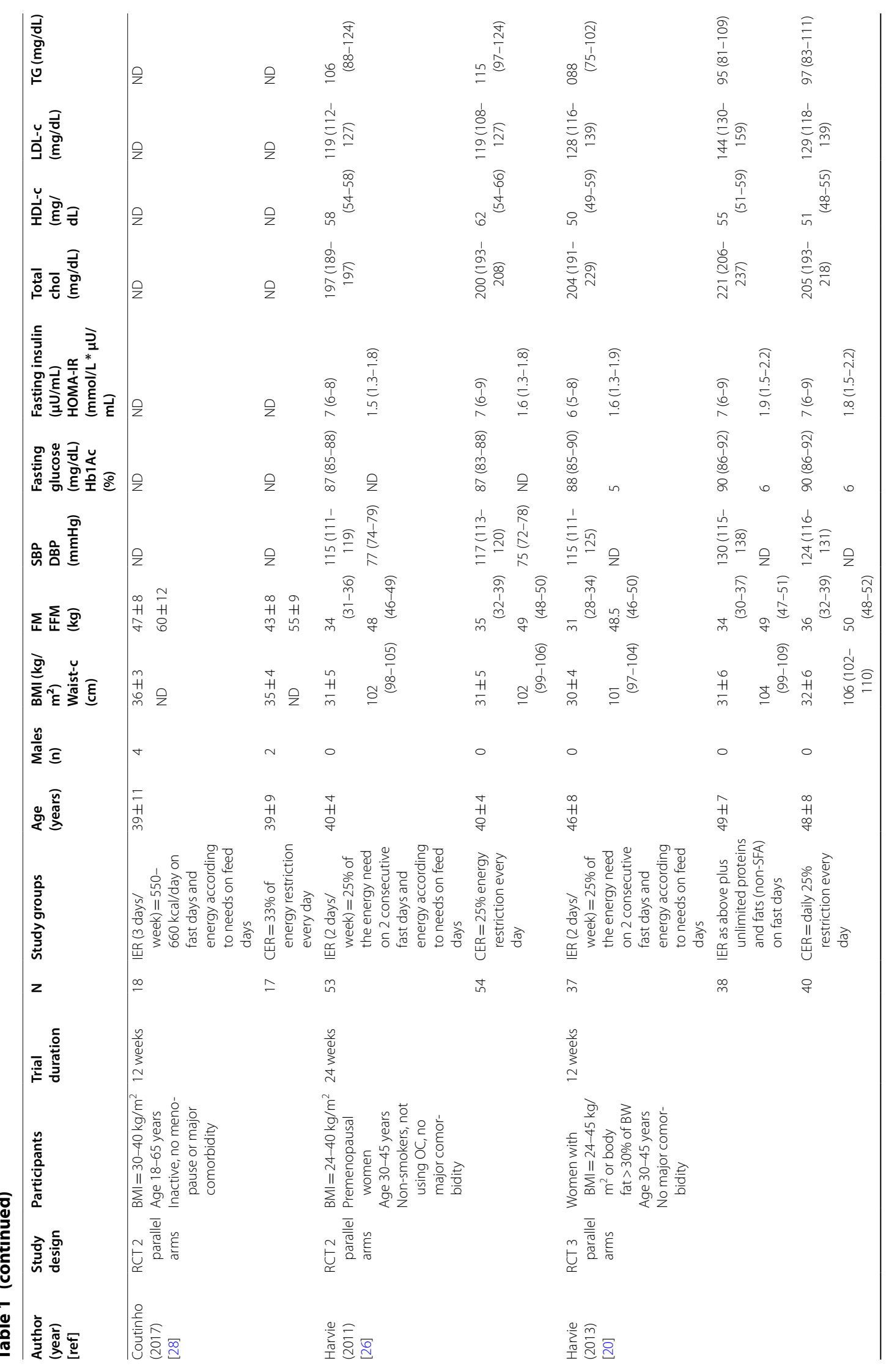




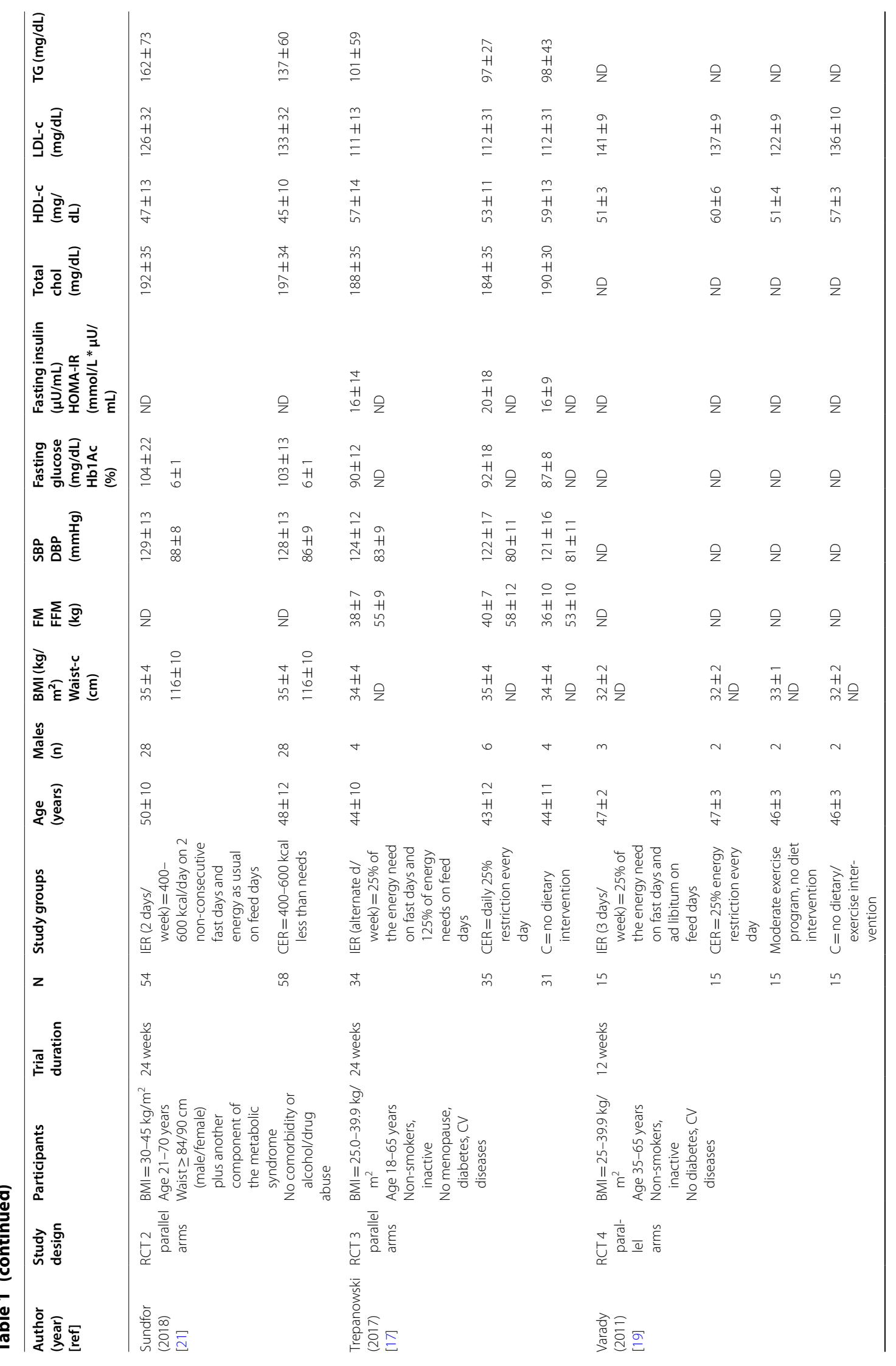




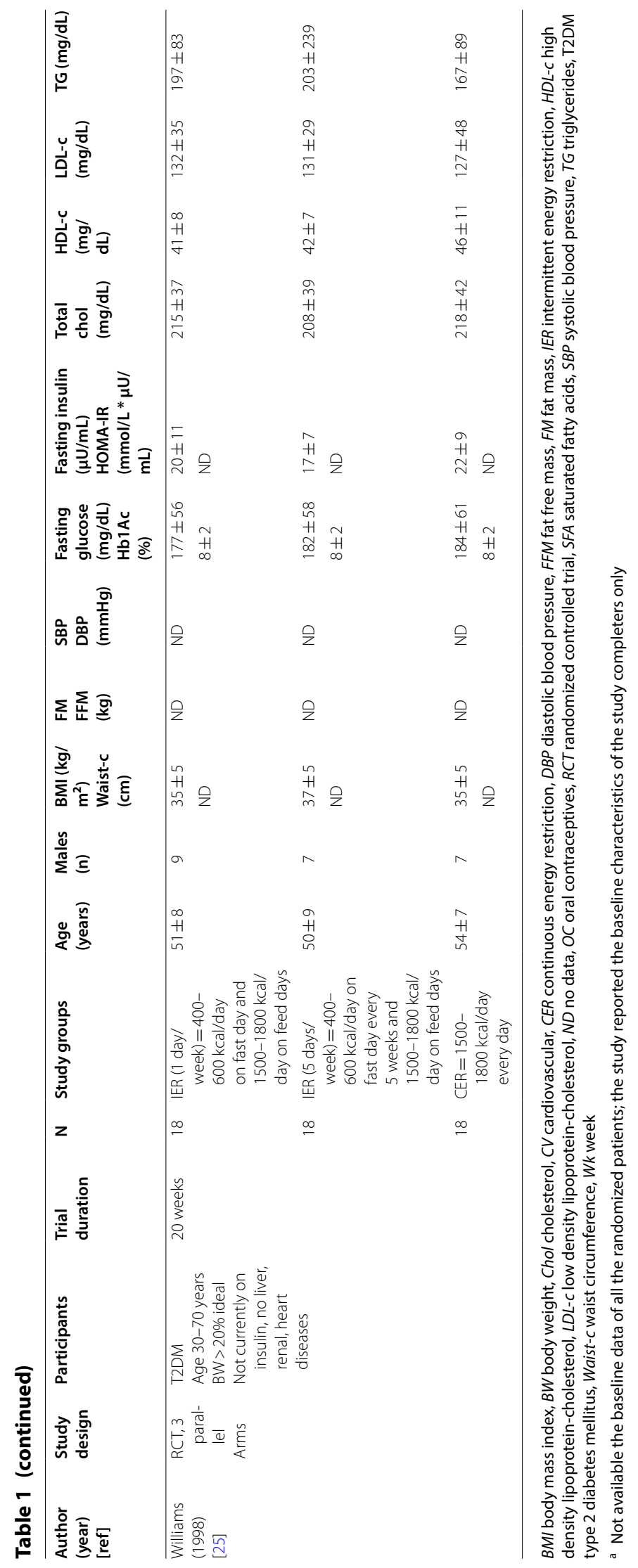




\section{Risk of bias assessment}

Some of the analyzed trials were characterized by the lack of information about the randomization procedures (Additional file 2). If blinding of participants was not feasible owing to the nature of the interventions, data about blinding of the personnel performing the laboratory or statistical analyses were always unknown, except for 1 study [20]. Dropouts were higher in the IER arms [17, 26, 28] or in the CER arms [20, 22, 24, 25], thus introducing a possible selection bias between-arms, but intention-to treat analyses were performed by all studies, except for 1 RCT [22], where data of the completers only have been reported. Finally, most trials appeared to be free of selective outcome reporting and of other sources of bias, apart from 1, where body weight at baseline was not reported [19].

\section{Meta-analysis}

All the outcomes of interest of this systematic review are reported in Additional file 3. Data synthesis was performed for the outcomes reported by $>3$ trials, therefore data relative to Si values were not pooled.

\section{Weight loss}

All RCTs reported weight loss in the IER arms during the intervention, ranging from $5.2 \%$ [19] of initial weight to $12.9 \%$ [28], while in the CER arms, changes ranged from 4.3\% [20] to $12.1 \%$ [28] (Additional file 3). Pooled data from random-effect analysis did not show a significant effect of IER on weight loss (WMD: $-0.61 \mathrm{~kg}, 95 \% \mathrm{CI}$ -1.70 to $0.47 ; \mathrm{p}=0.27$ ) (Fig. 2). The estimated effect on body weight did not change in the leave-one-out sensitivity analysis (data not shown).

Subgroup analyses based on the type of regimen (5:2 vs. other regimens) as well as on the dietary characteristics of the "feed" days of the IER interventions (ad libitum vs. balanced food intake) showed consistent results, as reported in Additional file 4. Analyses were repeated after the exclusion of the trial prescribing 5 consecutives "fast" days and then 1 "fast" day/week per 15 weeks [25], and the results did not change (WMD: $-0.36 \mathrm{~kg}, 95 \% \mathrm{CI}$ -1.48 to $0.77 ; \mathrm{p}=0.54)$. Finally, the $\mathrm{RCT}$ reporting the percent relative variations of the endpoints only [19] was included in the analyses, and the estimated effect size of weight change did not show any between-arms difference (WMD: -0.08 , 95\% CI -0.23 to 0.07 ; $\mathrm{p}=0.29$ ).

Similarly, the percent weight loss was similar in both arms (WMD: $-0.38 \%, 95 \% \mathrm{CI}-1.16$ to $0.40 ; \mathrm{p}=0.34$ ) and the results did not differ either in the subgroup analyses (Additional file 5) or in the leave-one-out sensitivity analysis.

\section{Other anthropometric measures}

Seven out of the 11 included RCTs reported changes in FM and FFM [17, 20, 22, 24, 26-28]. FM was measured by different methods: body impedance analysis (BIA) [20, 22]; dual X-ray absorptiometry (DXA) [17, 24, 27]; impedance [26]; air displacement plethysmography [28]. Pooled results showed no difference between-arms in FM (WMD: $-0.23 \mathrm{~kg}, 95 \% \mathrm{CI}-1.23$ to 0.77 ; $\mathrm{p}=0.66$ ) as well as in FFM (WMD: $-0.22 \mathrm{~kg}, 95 \% \mathrm{CI}-1.01$ to 0.56 ; $\mathrm{p}=0.58$ ), as shown in Additional file 6. Those results were consistent both at subgroup analyses and at sensitivity analyses. Five RCTs assessed waist circumference [20-23, 26] without showing any differences between arms (WMD: $-0.17 \mathrm{~cm}$; $95 \% \mathrm{CI}-1.74$ to $1.39 ; \mathrm{p}=0.83$ ).

\section{Cardiometabolic biomarkers}

Pooled data obtained from glucose, HbA1c, insulin and HOMA-IR are presented in Fig. $3 \mathrm{a}-\mathrm{d}$ respectively. Changes in fasting glucose and HbA1c values were reported respectively in $7[17,20-23,26,27]$ and $4[21$, 24-26] trials. Random-effect analysis showed no difference either on glucose (WMD: $-0.49 \mathrm{mg} / \mathrm{dL}, 95 \%$ CI -1.98 to $0.99 ; \mathrm{p}=0.51$ ) or HbA1c (WMD: $-0.02 \%$, $95 \% \mathrm{CI}-0.10$ to $0.06 ; \mathrm{p}=0.62$ ) changes in the IER when compared to CER arms with consistent results in subgroup/sensitivity analyses.

On the contrary, fasting insulin values were significantly reduced with IER (WMD $=-0.89 \mu \mathrm{U} / \mathrm{mL} ; 95 \%$ $\mathrm{CI}-1.56$ to $\left.-0.22 ; \mathrm{p}=0.009 ; \mathrm{I}^{2}=0 \%\right)$ and the estimated effect appeared robust in the leave-one-out sensitivity analysis (data not shown). Moreover, subgroup analyses showed that the 5:2 regimens were associated with increased insulin reductions (WMD: $-0.99 \mu \mathrm{U} / \mathrm{mL}$; 95\% CI -1.67 to $-0.30 ; \mathrm{p}=0.005 ; \mathrm{I}^{2}=0$ ) (Additional file 7 ). All the RCTs evaluating fasting insulin values included a balanced energy regimen for the "feed" days. HOMAIR values were reduced, though not significantly, in the IER regimens $(\mathrm{WMD}=-0.15 \mathrm{mmol} / \mathrm{L} \times \mu \mathrm{U} / \mathrm{mL} ; 95 \% \mathrm{CI}$ -0.33 to $0.02 ; \mathrm{p}=0.09)$.

Only 1 RCT evaluated insulin sensitivity $(\mathrm{Si})$ by a frequently sampled intravenous glucose tolerance [21], without between-arms differences.

Pooled data obtained from 8 RCTs [17, 20-23, 25-27] did not show any significant effect of IER on triglyceride concentrations (WMD: - $3.11 \mathrm{mg} / \mathrm{dL}, 95 \% \mathrm{CI}$ - 9.76 to $3.54 ; \mathrm{p}=0.36$ ) (Fig. 4a). However, subgroup analyses showed a slightly significant triglyceride reduction in the IER arms employing other fasting regimens (WMD $=-14.4 \mathrm{mg} / \mathrm{dL} 95 \% \mathrm{CI}-28.6$ to -0.23 ; $\left.\mathrm{p}=0.046 ; \mathrm{I}^{2}=0 \%\right)$. Characteristics of the "feed" days were not associated with differences in triglyceride changes (Additional file 8). HDL-cholesterol levels increased after IER regimens, albeit not significantly $(W M D=1.72 \mathrm{mg} /$ 
dL 95\% CI -0.20 to 3.63; $\mathrm{p}=0.07$ ) (Fig. 4c). Subgroup analysis revealed a significant HDL-cholesterol increase with a balanced diet on "feed" days (WMD $=2.88 \mathrm{mg} /$ dL 95\% CI 0.66 to 5.09; $\mathrm{p}=0.011 ; \mathrm{I}^{2}=0 \%$ ) compared with ad libitum eating (Additional file 9). No between-arm differences were found for total cholesterol and LDLcholesterol (Fig. 4b, d). Finally, changes in both SBP and DBP did not significantly differ between arms (Additional file 10).

\section{Publication bias}

We used the Egger's test for funnel plot asymmetry to detect a potential publication bias on reporting results on weight change. Test result $(\mathrm{p}=0.15)$ did not suggest any asymmetry in the funnel plot (Additional file 11).

\section{Safety}

No major adverse events were reported. Only 1 patient from the IER arm of the RCT supplying $0 \mathrm{kcal}$ during "fast" days developed gallbladder dyskinesia and underwent cholecystectomy after completing the study, but this event was reported to be unrelated to the intervention [27]. Minor physical or psychological adverse effects, such as lack of energy, headaches, feeling cold, constipation, bad breath, lack of concentration, bad temper, were reported in a minority of participants from the IER arms $(<20 \%)$ in a few studies $[20,21,23,26]$. On the other hand, hunger was reported in the first weeks by about half of participants to a 5:2 regimen in 1 trial, but this symptom improved over time [23].

\section{Discussion}

An intermittent regimen of energy restriction (at least 1 day/week) determined a loss in body weight and percent body weight similar to continuous (non-intermittent) energy restriction. Interestingly, a slight reduction in fasting insulin concentrations was evident with IER regimens employing 2 days/week "fast", but the clinical relevance of this result is uncertain.

\section{Effects of IER on weight loss and fat mass}

Most systematic reviews and meta-analyses demonstrated that IER regimens achieved comparable weight loss as CER regimens $[4,5,9]$, reporting an overall weight loss ranging from 4 to $8 \%[2,3,7,9]$, and a difference of $-4.14 \mathrm{~kg}$ to $+0.08 \mathrm{~kg}$ versus the comparator arms $[4,5$, 29]. Our results are in accordance, even if the trials previously included differed from ours, since we have included only RCTs with a at least 1 day/week and no more than 6 day/week of "fasting", and with an extremely low energy supply during the "fast" days. This latter choice derived from the idea of studying conditions simulating as much as possible a condition of fasting, whose benefits, proven by animal studies, seem to depend on the shift in metabolism from glucose utilization and fat synthesis/storage towards reduced insulin secretion and fat mobilization/ oxidation [30, 31].

There is no clear definition of IER, and intermittent regimens providing up to $800 \mathrm{kcal}[5,9]$, with $\geq 7$ "fast" days $[4,6,9,29]$, including time-restricted feeding [2, $6-8,32]$, with unlimited energy restriction as a comparator group [2, 3, 5-7], or not randomized controlled trials [2] have been included within previous reviews. We have taken care to define precise inclusion criteria to limit variability and increase the comparability among trials, and we have obtained a low heterogeneity.

It could be hypothesized that the very low caloric intake on "fast" days determined an overall lower caloric intake in the IER arms, which were therefore difficult to be compared with the CER arms. In the only RCT where water and calorie-free beverages were allowed in the "fast" days, a significant between-arms difference in energy intake was evident [27]; in two studies a between-arms difference of 300-400 kcal was observed $[22,23]$ while most RCTs reported a negligible between-arms difference ( $100 \mathrm{kcal})[17,20,21,25$, 26]. Consistently, our sensitivity and subgroup analyses did not find significant between-arms differences.

Furthermore, the percent weight loss was highly overlapping, and no apparent superiority of a dietary regimen was evident. Indeed, participants of the IER arms from all RCTs lost $\geq 5 \%$ of their initial weights, thus confirming the clinical usefulness of this approach at least in the short term, i.e. within 24 weeks.

Previous reviews reported a FM loss ranging from 4 to $7 \%$ [3] to $11-16 \%$ [2] in the IER arms, and the only meta-analysis evaluating this outcome reported a differential loss of $1.38 \mathrm{~kg}$ with respect to comparator arms [5]. We failed to find significant between-arms difference for this outcome, suggesting that such a regimen could be a valid, but not superior alternative to CER.

Intriguingly, participants to the IER regimens usually did not consume as much food in the "feed" days as to compensate for the caloric restriction of the "fast" days, thus suggesting that IER could reduce food intake even in the "feed" days, without compensatory overeating [6, $31]$. This finding was not confirmed by all studies [28, 33, 34]. Furthermore, adverse events were sometimes higher with the IER regimens $[20,21,26]$, and the participants reported stronger feelings of hunger [21, 23]. The compliance and adherence to the intervention diets was heterogeneous among trials, the attrition rate was often higher in the IER arms [17, 22, 24, 26, 31, 35], and the percentage of participants planning to continue with the dietary regimen beyond 6 months was lower in the IER 


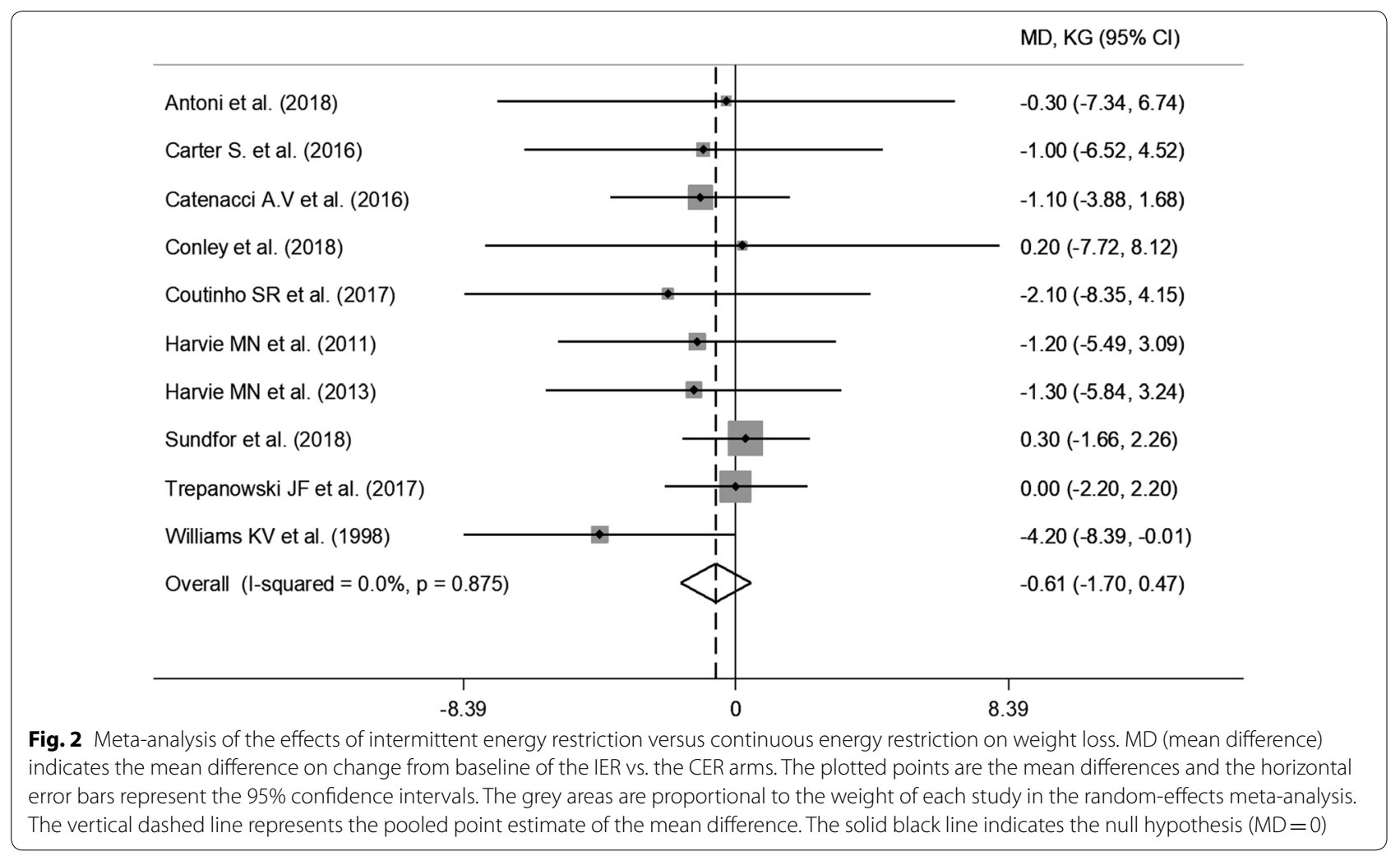

arms [26]. Overall, these data do not support the fact that IER is easier and more acceptable than CER to everyone. Moreover, the reduction in resting energy expenditure, i.e. the compensatory metabolic response which reduces the degree of weight loss, has been reported to be either reduced (favoring weight loss) [27, 36] or increased (attenuating weight loss) [22, 28] with IER regimens. Indeed, some studies suggest that IER evokes the same adaptive response as CER [6, 37].

The hypothesized benefits of IER, extensively studied in animal models, included the use of fats during severe energy restriction with preferential reduction of adipose mass, the stimulation of browning in white adipose tissue, increased insulin sensitivity, lowering of leptin and increased human growth hormone, ghrelin and adiponectin circulating levels, reduced inflammation and oxidative stress [30]. The trigger of adaptive cell response leading to enhanced ability to cope with stress, improved autophagy by sirtuin-1 activity stimulation, modification of apoptosis, increase of vascular endothelial growth factor expression in white adipose tissue, the action on the metabolism via Forkhead Box A genes, and reduction of advance glycation end-products might be all possible metabolic pathways explaining the beneficial effects of IER [7, 30, 38, 39]. In mice, IER determined metabolic improvements and weight loss as a consequence of a shift in the gut microbiota composition, leading to an increase in the production of acetate and lactate and to the selective upregulation of monocarboxylate transporter in beige adipose cells which stimulate beige fat thermogenesis [40]. At present, many of these adaptive mechanisms have been demonstrated in animal experimental models but not in humans, thus more research is still needed.

\section{Effects of IER on cardiometabolic markers}

IER regimens were associated with lower circulating insulin values; a significant reduction was evident for the 5:2 "fasting" regimen only. Indeed, two RCTs, both employing this regimen, determined the difference [20, 26]. Our data are in line with the results of a previous meta-analysis reporting a significantly higher reduction in fasting insulin $(-0.67 \mu \mathrm{U} / \mathrm{mL})$ in the IER arms [5]. The difference we found $(-0.89 \mu \mathrm{U} / \mathrm{mL})$ was statistically significant, but not clinically relevant, above all considering the fact that participants to the included RCTs were overweight/obese and therefore probably insulin-resistant individuals.

Our data synthesis on glucose, HOMA-IR, HbA1c showed no between-arms difference. We did not include patients with T2DM from 2 RCTS in the pooled analysis on fasting glucose, since most participants were on hypoglycemic drugs and their glycemic values would be 


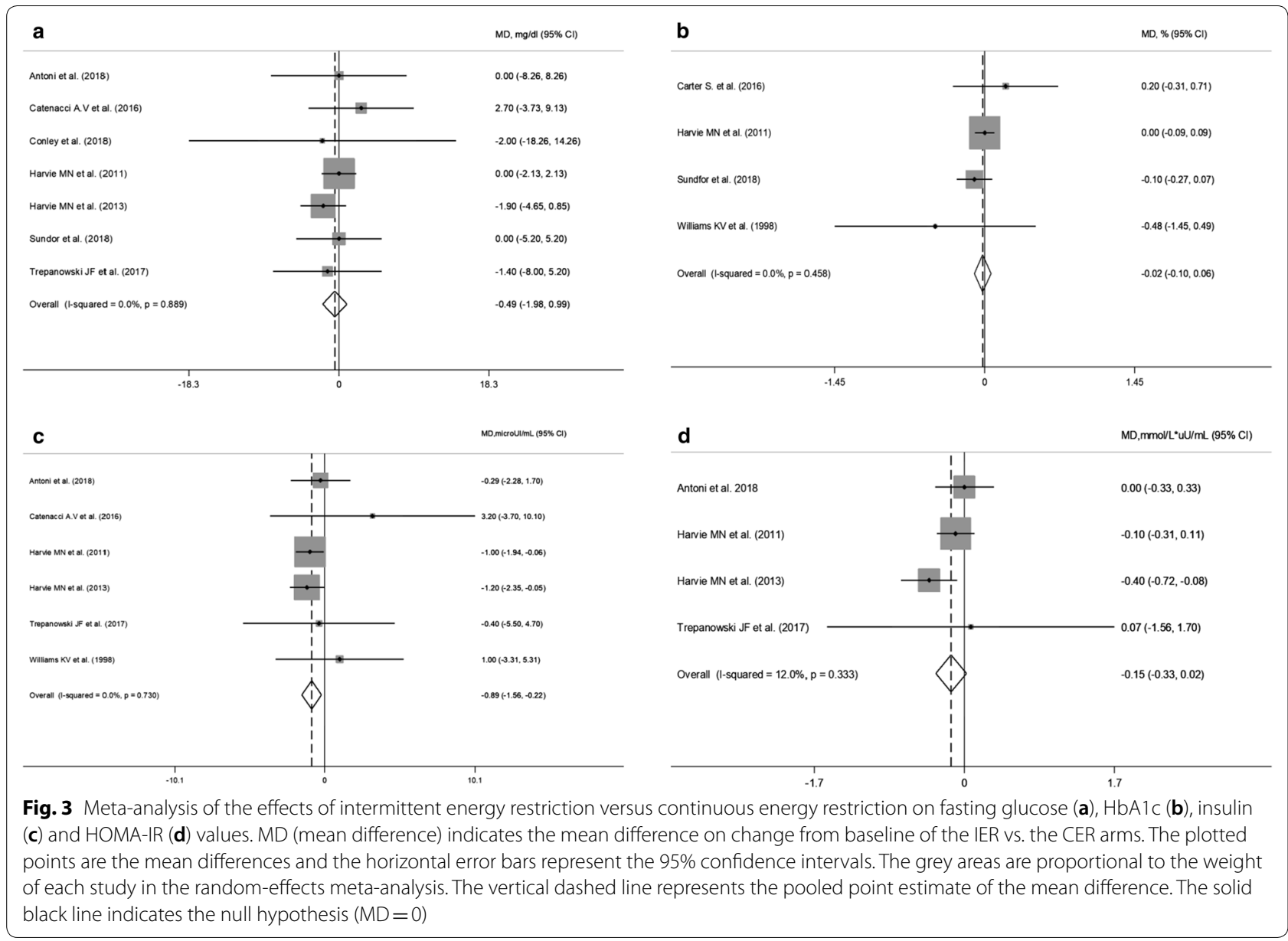

certainly influenced by the treatment [24, 25]. Highly contrasting human studies are available about the benefits of IER on glucose metabolism and insulin sensitivity $[3,6,31]$, contrarily to animal studies strongly suggesting a benefit in T2DM prevention $[1,31]$. The improvements in glucose homeostasis might be therefore comparable to those obtained by continuous energy restrictions.

Our meta-analysis did not show significant betweenarms difference in lipid values and arterial blood pressure, with the exception of a small difference in subgroup analyses on triglyceride concentrations $(-14 \mathrm{mg} / \mathrm{dL})$ and HDL-cholesterol $(+2.88 \mathrm{mg} / \mathrm{dL})$, not meaningful from a clinical point of view. Most studies showed reduction in triglyceride levels between 15 and $42 \%$ in the IER arms [31, 41], and the only available meta-analysis reported a between-arms not significant difference of $2.65 \mathrm{mg} /$ $\mathrm{dL}$ [5]. Reduction in total cholesterol, LDL-cholesterol in the IER arms ranged respectively between $6-25 \%$, 7-32\%, with small effects on HDL-cholesterol [1, 31], and between-arms differences resulted not significant [5]. Intriguingly, a few studies reported that IER regimens determined an increase in LDL particle size [19, 42], and reduced post-prandial hypertriglyceridemia [22], thus potentially conferring cardio-protection, since the lower the LDL size, the higher the oxidizability and the susceptibility to arterial penetration, and higher post-prandial hyperlipemia is a marker of atherosclerosis progression. Furthermore, fasting can act on many enzymes implicated in lipid and lipoprotein metabolism [27]. However, all these reports need confirmation in larger human RCTs.

Similarly, data on arterial BP were controversial, with the majority of human studies reporting no differences between IER and CER regimens [1, 5, 31, 41]. Indeed, most of the published studies and RCTs included normotensive subjects at baseline, making it difficult to identify differences between-arms.

Therefore, unlike the very promising data on animals, evidence is not sufficiently robust to suggest the superiority of intermittent vs. continuous caloric restriction regimens on the main cardiovascular factors in humans. 


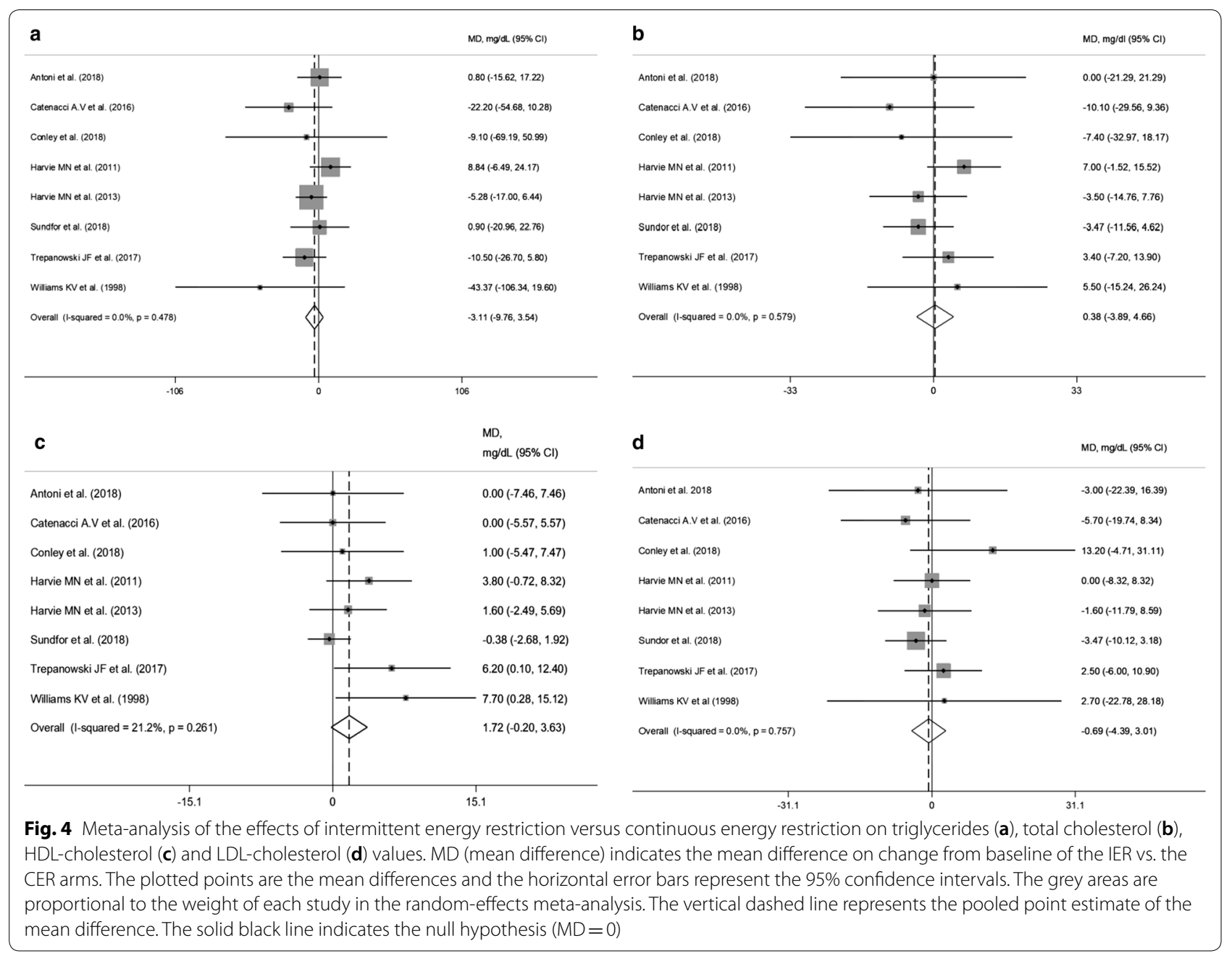

\section{Clinical implications}

Weight loss maintenance should be an integral component of the management of obesity, owing to the weight regain usually occurring with time. The 2 RCTs including longer follow-ups ( 24 months) did not find betweenarms differences in weight loss maintenance [17, 27]. Studies with longer follow-ups, evaluating the longterm sustainability, adherence to, and safety of IER regimens are needed. Furthermore, no RCT evaluated hard endpoints, such as cardiovascular outcomes or T2DM incidence. Two observational cohort studies found that fasting was associated with a lower prevalence of coronary artery diseases or diabetes diagnosis but are limited by a lack of a comprehensive dietary history and many potential bias $[43,44]$. It could be hypothesized that IER regimens should be proposed in clinical practice, since it is possible that some individuals find easier to reduce their energy intakes for 1 or more days per week, rather than every day. It is well known that a single diet fit not all, and in the choice of the individual's tailored regimen,
IER strategies should be considered by health care professionals. In this way, data on the feasibility of these regimens in "real life" would be obtained.

\section{Strengths and limitations}

This is, to our knowledge, the largest and updated metaanalysis on the effects of IER on weight loss and multiple metabolic outcomes, setting strict inclusion criteria to increase comparability among studies.

The high variability among the RCTs in the feeding protocols, the limited follow-up, the small sample sizes, the high drop-out rates potentially leading to selection bias, the limited reporting of adverse events and blinding of investigators about arm allocation, or other methodological problems are all limitations to be considered. Finally, most studies were performed by the same authors and the majority of subjects included were adult healthy women, thus limiting the generalizability of the results. 


\section{Conclusion}

In overweight/obese adults, IER is as effective as CER for promoting weight loss and metabolic improvements in the short term. Further long-term investigations are needed to draw definitive conclusions.

\section{Additional files}

\section{Additional file 1. Electronic search strategy.}

Additional file 2. Risk of bias assessment in the trials included in the systematic review.

Additional file 3. Changes in outcomes at the end of the trials.

Additional file 4. Subgroup analysis of weight loss based on the type of regimen (a) and on dietary characteristics of the "feed" days (b).

Additional file 5. Percent weight loss (a) and subgroup analysis of percent weight loss based on the type of regimen (b) and dietary characteristics of the "feed" days (c).

Additional file 6. Meta-analysis of the effects of intermittent energy restriction versus continuous energy restriction on body composition (a) and waist circumference (b).

Additional file 7. Subgroup analysis of fasting insulin based on the type of regimen.

Additional file 8. Subgroup analysis of triglycerides based on the type of regimen (a) and dietary characteristics of the "feed" days (b).

Additional file 9. Subgroup analysis of HDL-cholesterol based on the type of regimen (a) and dietary characteristics of the "feed" days (b).

Additional file 10. Meta-analysis of the effects of intermittent energy restriction versus continuous energy restriction on systolic blood pressure (SBP) (a) and diastolic blood pressure (DBP) (b).

Additional file 11. Funnel plot for publication bias detection on weight loss changes.

\section{Abbreviations}

BIA: body impedance analysis; BMI: body mass index; BP: blood pressure; CER: continuous energy restriction; $\mathrm{Cl}$ : confidence interval; CINAHL: Cumulative Index to Nursing and Allied Health Literature; DBP: diastolic blood pressure; DXA: dual X-ray absorptiometry; FFM: fat free mass; FM: fat mass; HbA1c: glycated hemoglobin A1c; HOMA-IR: Homeostasis Model Assessment-Insulin Resistance; IER: intermittent energy restriction; MeSH: medical subject headings; RCTs: randomized controlled trials; Si: insulin-sensitivity index; SBP: systolic blood pressure; T2DM: type 2 diabetes mellitus; WMD: weight mean difference.

\section{Authors' contributions}

IC participated in the conception and design of the study, data collection and revision, interpretation of the findings of the study, manuscript writing and revision. AE participated in the data analysis, interpretation of the findings, manuscript writing and revision. VP, GC participated in the data analysis, interpretation of the findings, and manuscript revision. LSo, LSa, FP, FC, EG participated in the interpretation of the findings, and manuscript revision. SB participated in the conception and design of the study, data collection and revision, manuscript writing and revision. All authors read and approved the final manuscript.

\section{Author details}

${ }^{1}$ Interuniversity Center for Obesity and Eating Disorders, Department of Medicine and Surgery, Federico II University Hospital, Pansini, 5, Naples 80131, Italy. ${ }^{2}$ Unit of Clinical Epidemiology, CPO, "Città della Salute e della Scienza" Hospital of Turin, Turin, Italy. ${ }^{3}$ Department of Medical Sciences, University of Turin, c.so AM Dogliotti 14, 10126 Turin, Italy. ${ }^{4}$ Department of Health Sciences, University of Milan, Milan, Italy.
Acknowledgements

Not applicable.

\section{Competing interests}

The authors declare that they have no competing interests.

Availability of data and materials

All data analyzed during this study are included in this published article.

Consent for publication

Not applicable.

Ethics approval and consent to participate

Not applicable.

Funding

Not applicable.

\section{Publisher's Note}

Springer Nature remains neutral with regard to jurisdictional claims in published maps and institutional affiliations.

Received: 31 October 2018 Accepted: 14 December 2018

Published online: 24 December 2018

\section{References}

1. Varady KA, Hellerstein MK. Alternate-day fasting and chronic disease prevention: a review of human and animal trials. Am J Clin Nutr. 2007;86:7.

2. Varady KA. Intermittent versus daily calorie restriction: which diet regimen is more effective for weight loss? Obes Rev. 2011;12:593.

3. Barnosky AR, Hoddy KK, Unterman TG, Varady KA. Intermittent fasting vs daily calorie restriction for type 2 diabetes prevention: a review of human findings. Transl Res. 2014;164:302.

4. Headland M, Clifton PM, Carter S, Keogh JB. Weight-loss outcomes: a systematic review and meta-analysis of intermittent energy restriction trials lasting a minimum of 6 months. Nutrients. 2016;8:8.

5. Harris L, Hamilton S, Azevedo LB, Olajide J, De Brún C, Waller G, et al. Intermittent fasting interventions for treatment of overweight and obesity in adults: a systematic review and meta-analysis. JBI Database Syst Rev Implement Rep. 2018;16:507.

6. Seimon RV, Roekenes JA, Zibellini J, Zhu B, Gibson AA, Hills AP, et al. Do intermittent diets provide physiological benefits over continuous diets for weight loss? A systematic review of clinical trials. Mol Cell Endocrinol. 2015:418:153.

7. Horne BD, Muhlestein JB, Anderson JL. Health effects of intermittent fasting: hormesis or harm? A systematic review. Am J Clin Nutr. 2015;102:464

8. Varady KA. Impact of intermittent fasting on glucose homeostasis. Curr Opin Clin Nutr Metab Care. 2016;19:300.

9. Davis CS, Clarke RE, Coulter SN, et al. Intermittent energy restriction and weight loss: a systematic review. Eur J Clin Nutr. 2016;70:292.

10. Rothschild J, Hoddy KK, Jambazian P, Varady KA. Time-restricted feeding and risk of metabolic disease: a review of human and animal studies. Nutr Rev. 2014:72:308.

11. Patterson RE, Laughlin GA, LaCroix AZ, Hartman SJ, Natarajan L, Senger CM, et al. Intermittent fasting and human metabolic health. J Acad Nutr Diet. 2015;115:1203.

12. Hutchison AT, Heilbronn LK. Metabolic impacts of altering meal frequency and timing — does when we eat matter? Biochimie. 2016;124:187.

13. Lantz H, Peltonen M, Agren L, Torgerson JS. Intermittent versus ondemand use of a very low calorie diet: a randomized 2-year clinical trial. J Intern Med. 2003;253:463

14. Moher D, Liberati A, Tetzlaff J, Group P. Preferred reporting items for systematic reviews and meta-analyses: the PRISMA statement. PLoS Med. 2009;6:e1000097. https://doi.org/10.1371/journal.pmed.1000097.

15. Higgins JP, Altman DG, Gotzsche PC, Jüni P, Moher D, Oxman AD, et al. The Cochrane Collaboration's tool for assessing risk of bias in randomised trials. BMJ. 2011;343:5928. 
16. Higgins JPT, Green S (editors). Cochrane handbook for systematic reviews of interventions version 5.1.0 [updated March 2011]. The Cochrane Collaboration; 2011.

17. Trepanowski JF, Kroeger CM, Barnosky A, Klempel MC, Bhutani S, Hoddy $\mathrm{KK}$, et al. Effect of alternate-day fasting on weight loss, weight maintenance, and cardioprotection among metabolically healthy obese adults: a randomized clinical trial. JAMA Intern Med. 2017;177:930.

18. Higgins J, Thompson SG, Deeks JJ, Altman DG. Measuring inconsistency in meta-analyses. Br Med J. 2003;327:557.

19. Varady KA, Bhutani S, Klempel MC, Kroeger CM. Comparison of effects of diet versus exercise weight loss regimens on LDL and HDL particle size in obese adults. Lipids Health Dis. 2011;10:119.

20. Harvie M, Wright C, Pegington M, McMullan D, Mitchell E, Martin B, et al. The effect of intermittent energy and carbohydrate restriction $v$. daily energy restriction on weight loss and metabolic disease risk markers in overweight women. Br J Nutr. 2013;110:1534.

21. Sundfør TM, Svendsen M, Tonstad S. Effect of intermittent versus continuous energy restriction on weight loss, maintenance and cardiometabolic risk: a randomized 1-year trial. Nutr Metab Cardiovasc Dis. 2018;28:698.

22. Antoni $\mathrm{R}$, Johnston $\mathrm{KL}$, Collins $\mathrm{AL}$, Robertson MD. Intermittent v. continuous energy restriction: differential effects on postprandial glucose and lipid metabolism following matched weight loss in overweight/obese participants. Br J Nutr. 2018;119:507.

23. Conley M, Le Fevre L, Haywood C, Proietto J. Is two days of intermittent energy restriction per week a feasible weight loss approach in obese males? A randomised pilot study. Nutr Diet. 2018;75:65.

24. Carter S, Clifton PM, Keogh JB. The effects of intermittent compared to continuous energy restriction on glycaemic control in type 2 diabetes; a pragmatic pilot trial. Diab Res Clin Pract. 2016;122:106.

25. Williams KV, Mullen ML, Kelley DE, Wing RR. The effect of short periods of caloric restriction on weight loss and glycemic control in type 2 diabetes. Diab Care. 1998;21:2.

26. Harvie MN, Pegington M, Mattson MP, Frystyk J, Dillon B, Evans G, et al. The effects of intermittent or continuous energy restriction on weight loss and metabolic disease risk markers: a randomized trial in young overweight women. Int J Obes. 2011;35:714

27. Catenacci VA, Pan Z, Ostendorf D, Brannon S, Gozansky WS, Mattson $\mathrm{MP}$, et al. A randomized pilot study comparing zero-calorie alternateday fasting to daily caloric restriction in adults with obesity. Obesity. 2016:24:1874

28. Coutinho SR, Halset EH, Gåsbakk S, Rehfeld JF, Kulseng B, Truby H, et al. Compensatory mechanisms activated with intermittent energy restriction: a randomized control trial. Clin Nutr. 2018;37:815.

29. Harris L, McGarty A, Hutchison L, Ells L, Hankey C. Short-term intermittent energy restriction interventions for weight management: a systematic review and meta-analysis. Obes Rev. 2018;19:1.
30. Longo VD, Mattson MP. Fasting: molecular mechanisms and clinical applications. Cell Metab. 2014;19:181.

31. Anton SD, Moehl K, Donahoo WT, Marosi K, Lee SA, Mainous AG 3rd, et al. Flipping the metabolic switch: understanding and applying the health benefits of fasting. Obesity. 2018;26:254.

32. Santos HO, Macedo RCO. Impact of intermittent fasting on the lipid profile: assessment associated with diet and weight loss. Clin Nutr ESPEN. 2018;24:14

33. Heilbronn LK, Smith SR, Martin CK, Anton SD, Ravussin E. Alternate-day fasting in nonobese subjects: effects on body weight, body composition, and energy metabolism. Am J Clin Nutr. 2005;81:69.

34. Arguin H, Dionne IJ, Sénéchal M, Bouchard DR, Carpentier AC, Ardilouze $J \mathrm{~L}$, et al. Short- and long-term effects of continuous versus intermittent restrictive diet approaches on body composition and the metabolic profile in overweight and obese postmenopausal women: a pilot study. Menopause. 2012;19:870

35. Horne BD. Is periodic fasting really good for reducing cardiovascular risk and improving heart health? Fut Cardiol. 2011;7:721.

36. Byrne NM, Sainsbury A, King NA, Hills AP, Wood RE. Intermittent energy restriction improves weight loss efficiency in obese men: the MATADOR study. Int J Obes. 2018;42:129.

37. Harvie M, Howell A. Potential benefits and harms of intermittent energy restriction and intermittent fasting amongst obese, overweight and normal weight subjects - a narrative review of human and animal evidence. Behav Sci. 2017;19:7.

38. Golbidi S, Daiber A, Korac B, Li H, Essop MF, Laher I. Health benefits of fasting and caloric restriction. Curr Diab Rep. 2017;17:123.

39. Kim KH, Kim YH, Son JE, Lee JH, Kim S, Choe MS, et al. Intermittent fasting promotes adipose thermogenesis and metabolic homeostasis via VEGFmediated alternative activation of macrophage. Cell Res. 2017;27:1309.

40. Li G, Xie C, Lu S, Tian Y, Li L, Patel D, et al. Intermittent fasting promotes white adipose browning and decreases obesity by shaping the gut microbiota. Cell Metab. 2017;26:672.

41. Johnstone A. Fasting for weight loss: an effective strategy or latest dieting trend? Int J Obes. 2015;39:727.

42. Klempel MC, Kroeger CM, Varady KA. Alternate day fasting increases LDL particle size independently of dietary fat content in obese humans. Eur J Clin Nutr. 2013;67:783.

43. Horne BD, May HT, Anderson JL, Kfoury AG, Bailey BM, McClure BS, Intermountain Heart Collaborative Study, et al. Usefulness of routine periodic fasting to lower risk of coronary artery disease in patients undergoing coronary angiography. Am J Cardiol. 2008;102:814.

44. Horne BD, Muhlestein JB, May HT, Carlquist JF, Lappé DL, Bair TL, Intermountain Heart Collaborative Study Group, et al. Relation of routine, periodic fasting to risk of diabetes mellitus, and coronary artery disease in patients undergoing coronary angiography. Am J Cardiol. 2012;109:1558.
Ready to submit your research? Choose BMC and benefit from:

- fast, convenient online submission

- thorough peer review by experienced researchers in your field

- rapid publication on acceptance

- support for research data, including large and complex data types

- gold Open Access which fosters wider collaboration and increased citations

- maximum visibility for your research: over $100 \mathrm{M}$ website views per year

At BMC, research is always in progress.

Learn more biomedcentral.com/submissions 